\title{
Research on the Influence Mechanism of Feedback in the Learning Process of Motor Skills
}

\author{
Fan ZHANG ${ }^{1,2, *}$, Bo ZHOU ${ }^{1}$ and Chang-zhi $\mathrm{JIA}^{1}$ \\ ${ }^{1}$ Department of Police Skills and Tactics, Nanjing Forest Police College, Nanjing, China \\ ${ }^{2}$ Sports Science Postdoctoral Programme, Nanjing Normal University, Nanjing, China \\ ${ }^{*}$ Corresponding author
}

Keywords: Influence Mechanism; Feedback; Motor Skill Learning; Learning Process.

\begin{abstract}
The purpose of this study is to explore the mechanism of feedback in the learning process of motor skills through literature review. In the process of feedback to the implementation of action skill learning, the key is whether an additional feedback arrangement can trigger an action skill learner to carry out error detection through the information of internal feedback. Therefore, this study focuses on the frequency, timing and error estimation of feedback to explore the mechanism of feedback in learning motor skills. The paper suggests that the provision of augmented feedback should consider its significance to learners so that learners can use feedback information to generate appropriate reflections. Feedback should be provided in such a way as to prevent learners from relying on extrinsic feedback and to trigger learners' information processing for intrinsic feedback.
\end{abstract}

\section{Introduction}

Feedback is one of the main topics in the research field of motor skill learning. After an action is executed, people can understand the process and results of the action through information provided by various sensory perceptions or additional information provided by the outside world [1,2]. Therefore, feedback can be divided into intrinsic feedback, also called intrinsic feedback, and extrinsic feedback, also called augmented feedback; extrinsic feedback is divided into knowledge of performance (KP) and knowledge of results (KR) based on what it provides. Magill pointed out in view of the information value of intrinsic feedback and extrinsic feedback that when intrinsic feedback cannot provide sufficient information, the demand of action executors for extrinsic feedback in terms of information increases accordingly [3]. Hence, the discussion of feedback must involve the characteristics of action itself in terms of intrinsic feedback and the ability of learners to perceive intrinsic feedback information, which means that the provision of augmented feedback is not absolutely necessary. However, the acquisition of feedback has the information function of providing movement correction and control after movement execution, stimulating learning motivation, and enhancing the connection function of correct movement stimulus and response, thus affecting movement displaying and learning.

\section{Research Progress}

The early theory of motor skill learning thinks that augmented feedback should be provided after each exercise so that learners can compare the movement parameters with the movement results of the sensation and perception. If feedback is not provided after each exercise, learners can only estimate the results based on relatively inaccurate subjective errors, thus leading to weakening of the movement representation or schema theory, and reducing the effect of motor skill learning. However, this view on the positive effect of motor skill learning lies in the role of movement performance rather than actual motor skill learning. The proposal of the guidance hypothesis has a great influence on the feedback study of motor skill learning. The viewpoint of distinguishing movement performance from learning leads to a new theoretical viewpoint in the following researches. High frequency feedback is only a variable of action performance. After each exercise, providing feedback guidance is beneficial 
to action performance, but it will make learners rely on extrinsic feedback. According to the viewpoint of error estimation, providing feedback after each exercise will reduce learners' experience in error detection based on intrinsic feedback and reduce the degree of cognitive effort. Therefore, the research on KR feedback no longer regards acquisition phase's performance that is easily guided by feedback as motor skill learning, but evaluates the learning effect by maintaining the design of tests or transfer tests. In this regard, Lee, Swinnen, and Serrien point out from the perspective of motor skill learning that if the learning efficiency of motor skill is improved, it may be necessary to guide learners' cognitive efforts so as to improve their processing ability of intrinsic feedback information to produce effective motor skill learning effect [4].

\section{Influence Mechanism}

\subsection{Relative Frequency Feedback}

The most direct point of view that affect that feedback arrangement after the G hypothesis is put forward directly through the research on reducing the relative frequency [5]. The research proves that although the decrease in the frequency of augmented feedback during the acquisition period is not necessarily beneficial to the performance of movement, the group that maintain the lower feedback arrangement in the test is superior to the group of feedback arrangement with higher frequency in the effect of learning action skills and the ability of error detection, whether it is during observation learning or actual practice. In response, Young and Schmidt pointed out that under the training conditions provided by high-frequency extrinsic feedback, learners can expect the provision of extrinsic feedback and rely on extrinsic feedback information for effective action correction [6].

On the contrary, if the frequency provided by extrinsic feedback is reduced, learners cannot use high-frequency extrinsic feedback information as the basis for action correction so they take the intrinsic feedback information as the basis for the action correction. In addition to causing improper correction of action performance, reducing high-frequency extrinsic feedback indirectly encourage learners to make wrong estimation, which is beneficial to the learning effect of action skills. Based on the above research and $\mathrm{G}$ hypothesis, providing less extrinsic feedback information will reduce learners' dependence on extrinsic feedback during the acquisition period and instead use intrinsic feedback information to show better performance in the retention test. Beside directly testing the effect of $\mathrm{G}$ hypothesis on motor skill learning in the way of reducing relative frequency, subsequent studies cover summary KR, faded feedback, self-controlled feedback, or bandwidth feedback that provides feedback based on the error value of movement performance. The theoretical basis provided by various kinds of feedback is not the same, but they are all influenced by G hypothesis. That is to say, they all focus on controlling the immediacy and frequency of feedback so as to increase learners' cognitive involvement and further reduce learners' dependence on extrinsic feedback.

\subsection{KR-delay}

KR-delay's provision after exercise has a better learning effect than providing KR immediately after exercise. Its mechanism is to enable learners to use intrinsic feedback to make wrong estimation and build up the action schema. In addition, according to the blocking hypothesis's view on the timing of providing feedback, if feedback is provided immediately after practice, learners will switch to using the information provided by extrinsic feedback directly, which interferes with cognitive activities using intrinsic feedback for performance, hinders their information processing, and reduces the effect of action skill learning. Park, Shea, and Wright examine the effect of concurrent feedback and terminal feedback on learning force-controlled actions [7]. The result shows that the effect of concurrent feedback on action skill learning is relatively insignificant, and it is inferred that concurrent feedback provides guidance for learners to ignore the effect of intrinsic feedback.

Moreover, the summary feedback is a feedback arrangement design influenced by the G hypothesis and the blocking hypothesis. By providing feedback after a series of exercises, the feedback providing process involves the delay interval in the time series. In addition to the effect on memory, KR-delay 
can give learners time to process intrinsic feedback information and develop error detection, thus leading to better retention effect. Previous studies have confirmed that summary feedback is more beneficial to motor skill learning than feedback provided immediately. The results of the study that provide feedback after a single exercise also support the view that KR should be provided immediately after exercise so that learners do not have the feedback arrangement method to exercise the error estimation, which is not conducive to the cognitive view of the learning effect of motor skills.

\subsection{Error Estimation}

The G hypothesis's view on feedback arrangement advocates avoiding learners' dependence on extrinsic feedback information and emphasizes the effect of intrinsic feedback information processing in action skill learning, which leads to the function of error estimation in action skill learning. The error estimation must use the intrinsic feedback information to compare the difference between the action parameters and subjective perception, and then compare with the actual action performance provided by the extrinsic feedback, so as to correct the subsequent performance of the movement and generate a stable action schema. Schmidt and Lee put forward the concept of efference copy for action control [8]. When a person issues an action command during an active action, the nervous system simultaneously generates duplicated information and transmits it to the brain, which can be used as the evaluation of the correct reference of actions. This signal represents the feeling about subjective and correct action execution.

After comparing the action performance with the expected action target through error estimation and comparing with KR information, people can carry out the modified feed forward representation of the next action execution parameter according to the outgoing copy. This is the basic discussion of error estimation on the basis of action control. Both in the learning process of fast action or slow action, people use the intrinsic feedback generated by action execution to carry out error detection on the error of action performance. The action executor can not only correct current action performance in the closed-loop feedback system, but also correct the next action performance. From the perspective of schema theory, error estimation is considered as the basis for error correction, and in order to confirm the important mechanism of schema learning, from the perspective of G hypothesis, KR with high frequency or immediacy can lead to better action performance, but it is not conducive to the effect of action skill learning because learners lack it.

Relevant research confirmed that in the retention test that does not provide KR, learners are unable to show good performance due to their lack of error detection ability. Previous studies on relative frequency feedback and error estimation in motor skill learning, have confirmed that the acquisition period requires learners to make error estimation after practice, which can avoid the negative impact of high-frequency feedback on motor skill learning. Guadagnoli and Kohl examine the effect of feedback on motor skill learning which provides frequency and error estimation frequency [9]. The research results show that after each exercise, requiring learners to make error estimation and match with $100 \% \mathrm{KR}$ can produce the best motor skill learning effect. The above research shows that error estimation can not only prevent learners from relying on high-frequency extrinsic feedback, but also promote more opportunities for comparison of intrinsic and extrinsic feedback, so as to improve the level of information processing and the cultivation of learners' error detection ability [10, 11]. Thus, learners can use intrinsic feedback information to complete the learning of action behaviors without relying on extrinsic feedback to provide information.

\section{Summary}

To sum up, the provision of augmented feedback should consider its significance to learners so that learners can use feedback information to generate appropriate reflections. Feedback should be provided in such a way as to prevent learners from relying on extrinsic feedback and to trigger learners' information processing for intrinsic feedback. In addition, the timing for providing feedback 
should not be too close to the execution of the action, which may distract the attention to the extrinsic feedback information; learners should be provided with time to process the intrinsic feedback information. If the frequency of feedback provision is too high, learners will expect the feedback provision after practice and ignore the intrinsic feedback information. Therefore, appropriately reducing the frequency of feedback provision will help to avoid the negative effect of the expected extrinsic feedback information. Therefore, before receiving feedback, learners of motor skills should first make a wrong estimation of movement performance based on the intrinsic feedback. In addition, teachers should adjust the frequency of feedback provision, taking into consideration learners' ability and work difficulty.

\section{Acknowledgement}

This work was supported in part by the Project of the Fundamental Research Funds for the Central Universities under Grant LGZD201805, in part by Jiangsu University Philosophy and Social Sciences Research Fund Project under Grant 2017SJB0590, in part by the 13th Five-Year plan project of Jiangsu Education Science under Grant C-c/2018/01/11, in part by Nanjing Forest police College Teaching Reform Project under Grant ZD18104, in part by the Project of China Postdoctoral Science Foundation under Grant 2017M611849, in part by Jiangsu Qing Lan Project under Grant 2017, and in part by Pre-research project of Nanjing Forest police College under Grant LGY201603.

\section{References}

[1] Sadowski J, Mastalerz A, Niznikowski T. Benefits of bandwidth feedback in learning a complex gymnastic skill [J]. Journal of Human Kinetics, 2013, 37 (1): 183-193.

[2] Sanli E A, Patterson J T. Learning effects of self-controlled practice scheduling for children and adults: are the advantages different? [J]. Perceptual \& Motor Skills, 2013, 116 (3): 741.

[3] Magill R A, Chamberlin C J, Hall K G. Verbal knowledge of results as redundant information for learning an anticipation timing skill [J]. Human Movement Science, 1991, 10 (4): 485-507.

[4] Timothy D. Lee, Stephan P. Swinnen, Deborah J. Serrien. Cognitive Effort and Motor Learning [J]. 1994, 46 (3): 328-344.

[5] Lee T D, Wishart L R, Magill R A. Motor learning conundrums (and possible solutions) [J]. Quest, 2005, 57 (1): 67-78.

[6] Schmidt R A, Young D E, Swinnen S, et al. Summary knowledge of results for skill acquisition: support for the guidance hypothesis [J]. Journal of Experimental Psychology Learning Memory \& Cognition, 1989, 15 (2): 352.

[7] Jin-Hoon Park, Charles H. Shea, David L. Wright. Reduced-Frequency Concurrent and Terminal Feedback: A Test of the Guidance Hypothesis [J]. Journal of Motor Behavior, 2000, 32 (3): 287-296.

[8] Schmidt R A, Lee T D. Motor control and learning: a behavioral emphasis [M]. Human Kinetics, 1999.

[9] Guadagnoli M A, Kohl R M. Knowledge of results for motor learning: relationship between error estimation and knowledge of results frequency [J]. Journal of Motor Behavior, 2001, 33 (2): 217.

[10] Sadowski J, Mastalerz A, Niznikowski T. Benefits of bandwidth feedback in learning a complex gymnastic skill [J]. Journal of Human Kinetics, 2013, 37 (1): 183-193.

[11] Patterson J T, Carter M, Sanli E. Decreasing the proportion of self-control trials during the acquisition period does not compromise the learning advantages in a self-controlled context [J]. Research Quarterly for Exercise \& Sport, 2011, 82 (4): 624-633. 